\title{
El restaurador como intermediario en la intervención de arte contemporáneo: la toma de decisiones
}

\author{
Miriam Limón Gallegos \\ Alma Maythé Loza Barajas
}

E

I restaurador, además de intervenir, tiene como misión rescatar la mayor cantidad posible de información que arroja el objeto que se ha de restaurar, así como analizar la documentación existente relacionada con éste. El objeto no es otra cosa que un material cuya función, como "bien cultural", se reconoce por los valores estéticos, religiosos, históricos o económicos que le atribuye la sociedad o su propietario, en muchos casos sólo ceñidos a su antigüedad. Respecto de los objetos patrimoniales, con características materiales, formales y conceptuales tradicionales (o considerados históricos), el restaurador se limita forzosamente a conocer al autor por medio de investigaciones historiográficas o de la comparación con características de obras similares con las que cuenta. Para él/ella podría parecer innecesario tratar de indagar la relación existente entre el dueño actual de la obra y el artista, para determinar el curso del proceso de restauración. No obstante, por la propia investigación el restaurador establece una relación con el dueño, aunque en la mayoría de las ocasiones ésta se constriñe a conseguir información útil para establecer las condiciones de conservación; o bien, para estimular un interés tal que conduzca a una completa revalorización e identificación con la obra (Barbero 2008:17). Por esto, en el caso de muchas de las obras no contemporáneas el restaurador se ubica en una relación especialista-objeto en la que no tiene contacto directo con los involucrados y, en ocasiones, ni siquiera con sus dueños. En esta operación, donde se realiza un proceso de extracción de información lineal, el restaurador funciona meramente a manera de eslabón.

Ahora bien, para el caso del arte contemporáneo existen algunas consideraciones que inciden en el tratamiento de la obra, situación en la cual difieren ampliamente de las que es factible encontrar en obras de carácter tradicional. Así como en la restauración de este tipo de piezas cada una de ellas es un objeto único por sí mismo y, como indica Salvador Muñoz Viñas (2003: 84), para su intervención es necesario realizar un análisis profundo de sus características materiales, conceptuales, históricas y contextuales, para las de las obras creadas recientemente, debido al modo de comunicación del mensaje —ya que la materia que lo conforma está dotada de un alto grado de significación, 
es decir, sus materiales, su forma, aplicación y distribución dentro de ella reflejan la intencionalidad artísticacada componente cuenta con una carga de valores, depositados por el autor, que en ocasiones el espectador no asume con tanta facilidad (Scicolone 2002:18).

En la intervención de restauración de arte contemporáneo el restaurador actúa como punto de conexión entre los actores involucrados directamente con la obra. No debe olvidarse, sin embargo, que aquélla no es más que un punto mediador, por cuya causa se establecen relaciones, se mantienen conceptos y se crean sentimientos que es imposible desconocer o pasar por alto, y por ninguna razón el objeto puede prescindir de ellos.

Esta es la complejidad que el restaurador, a partir de establecerse como intermediario, debe entender claramente al abordar su intervención, pero no sólo ello, sino considerar todas las partes, saber comunicarse con ellas y, a través de un lenguaje común, buscar el mejor resultado para la conservación no sólo de la obra sino también del mensaje que comunica.

En la mayoría de ocasiones el restaurador toma las decisiones sobre el trabajo tanto con base en la aplicación de conceptos teóricos como en su experiencia práctica. Pero cuando se trata de obra contemporánea, que los usuarios de museos o sus dueños "consumen", y que como objeto consumible siempre mantiene una relación con sus espectadores y creadores, se vuelve fundamental realizar cuestionamientos en cuanto a los límites de la intervención, el papel del artista y la consideración de su opinión en todo momento, para, con base en ello, elaborar esta toma de decisiones que repercutirá en su conservación material y/o conceptual.

A continuación se muestra un gráfico del esquema planteado, en el que se observa, de izquierda a derecha, la relación entre el artista y el coleccionista, transmitida a través del restaurador, quien actúa — permítasenos insistir en ello-como intermediario por medio de la obra, con el fin de establecer las bases para la toma de decisiones de restauración de arte contemporáneo (Figura 1).

En esta toma de decisiones las partes o actores involucrados que se han de considerar son, de manera general:

- El artista: se caracteriza por la creación de obras con una doble polaridad, en la que se engloba tanto la materia como el concepto (Llamas 2006:174). Las obras que elabora tienen una intención artística, es decir, los materiales que ha elegido comportan una carga conceptual que no puede separarse de su sentido. Específicamente para el arte contemporáneo, algunos artistas se preocupan por la selección de los materiales, para lo que usan los de mejor calidad o incluso, con el fin de lograr la preservación de su trabajo, se apegan a un rigor académico - aunque en muchos otros casos no es así, ya que experimentan con otros, en busca de diferentes características expresivas, provocando la aparición de alteraciones en un corto periodo de tiempo-. Para el caso de los deterioros en las capas pictóricas de sus obras, hay quienes prefieren realizar las intervenciones de restauración ellos mismos, pues así mantienen la imagen original, mas en otros casos modifican en gran medida la composición de la pieza (Llamas 2009: 82-83).

- El coleccionista, o consumidor de la obra, es aquel experto o aficionado a las expresiones artísticas de uno o más autores que reconoce, antes de adquirir una determinada obra, su calidad material o conceptual. Aunque se trate de arte contemporáneo, en ocasiones no tiene contacto directo con el artista, ya que adquiere las piezas por conducto de intermediarios, y en muchas otras desconoce no sólo la composición material

\section{Artista}

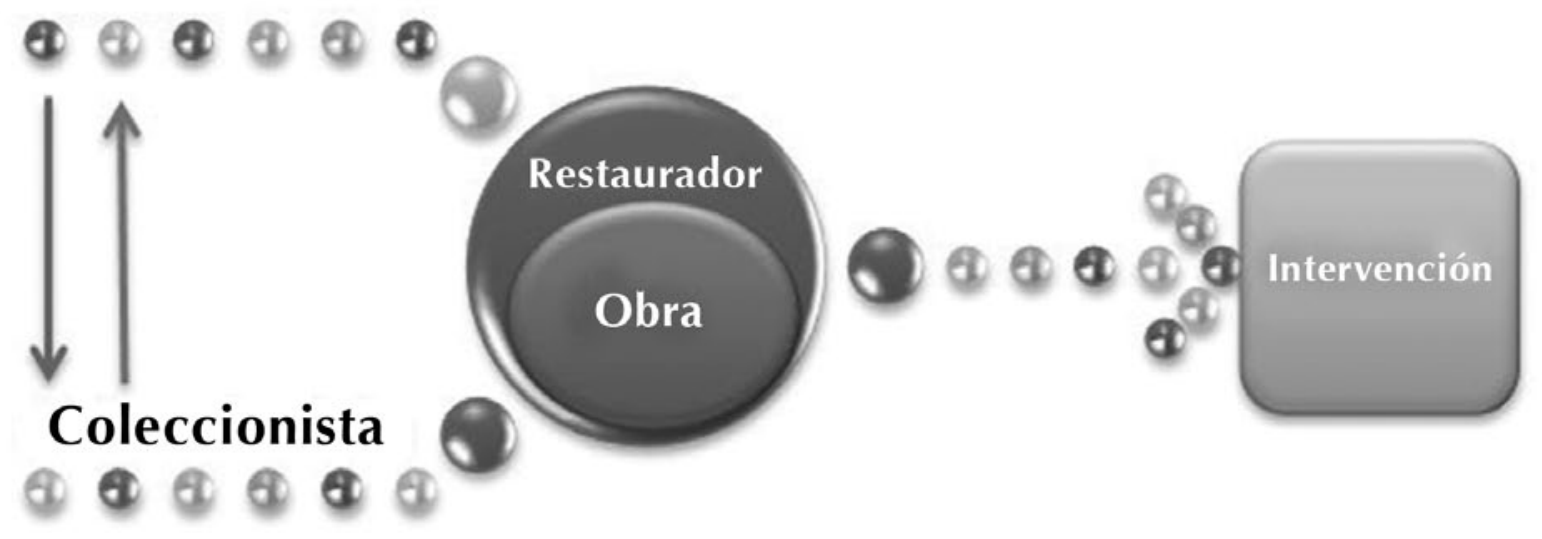

FIGURA 1. Esquema de diálogo de toma de decisiones para la intervención en el arte contemporáneo (Elaboración: Alma Maythé Loza Barajas, 2011). 
de la obra, sino incluso la intencionalidad del autor al haberla creado (Cauquelin 2002: 34).

- El restaurador es el especialista encargado de garantizar la persistencia de la obra para el futuro. Sus intervenciones, en el caso de arte contemporáneo, han de respetar ante todo el concepto que el autor asignó a la pieza sin privilegiar el material, ya que éste actúa sólo como conducto de los conceptos. Por esto el restaurador debe reconocer los valores implícitos de la pieza (Scicolone 2002:19). También ha de reconocer el aspecto documental de la obra, que actúa como un punto indispensable en la toma de decisiones, por lo que el carácter de la documentación debe ser exhaustivo, e incluso llegar a convertirse en un apoyo definitivo para la intervención. Las interrogantes que el restaurador se plantea —como, por ejemplo, hasta dónde están los límites del papel del artista en el desarrollo de propuestas de trabajo- son indispensables, y abrir un diálogo objetivo con los involucrados en la intervención de una determinada obra permite conocer datos que pueden llegar a definir un determinado tratamiento (Llamas 2009:75-76). Para conseguir la información proveniente de las partes involucradas, es necesario que se haga uso de herramientas precisas, como los métodos de entrevista, cuya finalidad estriba en la recolección de experiencias plásticas y conceptuales, además de los contextos y relaciones artísticos (Mata y Landa 2011:75), en el caso del artista y el coleccionista, respectivamente.
Con base en lo anteriormente dicho, el restaurador será capaz de crear un sistema de ideas en el que se involucran las intenciones artísticas, los criterios de intervención, las características materiales, las necesidades contextuales y las diferentes valoraciones sobre el objeto en cuestión de los agentes involucrados, con lo que, a través de una ruta metodológica de aproximación, se obtendrán las herramientas necesarias para la aplicación de acciones de conservación y restauración.

Para ejemplificar el sistema de diálogo planteado, aquí se expone el caso de la intervención de la pieza denominada Chamarra/retrato de Javier Campos Cabello, la cual requirió intervención de restauración durante su montaje de la exhibición Martha Pacheco. Exposición antológica, celebrada en el Museo de Arte de Zapopan (MAZ), dependiente de Ayuntamiento de la Ciudad de Zapopan, Jalisco (México) en septiembre del 2011. Debido a que se trataba de un óleo pintado sobre una prenda de vestir, mismo que poseía una capa de preparación de gesso comercial muy delgada, la manipulación de la pieza como objeto de arte generó problemas de adherencia en sus estratos pictóricos a lo largo del tiempo, manifestados en desprendimientos y craqueladuras. Así surgieron cuestionamientos respecto de su conservación y se planteó la posibilidad de intervención previa a su incorporación a la citada exposición.

El primer paso para las restauradoras fue el acercamiento con la directora del museo, Alicia Lozano, dueña de la pieza y compañera del fallecido artista, Javier

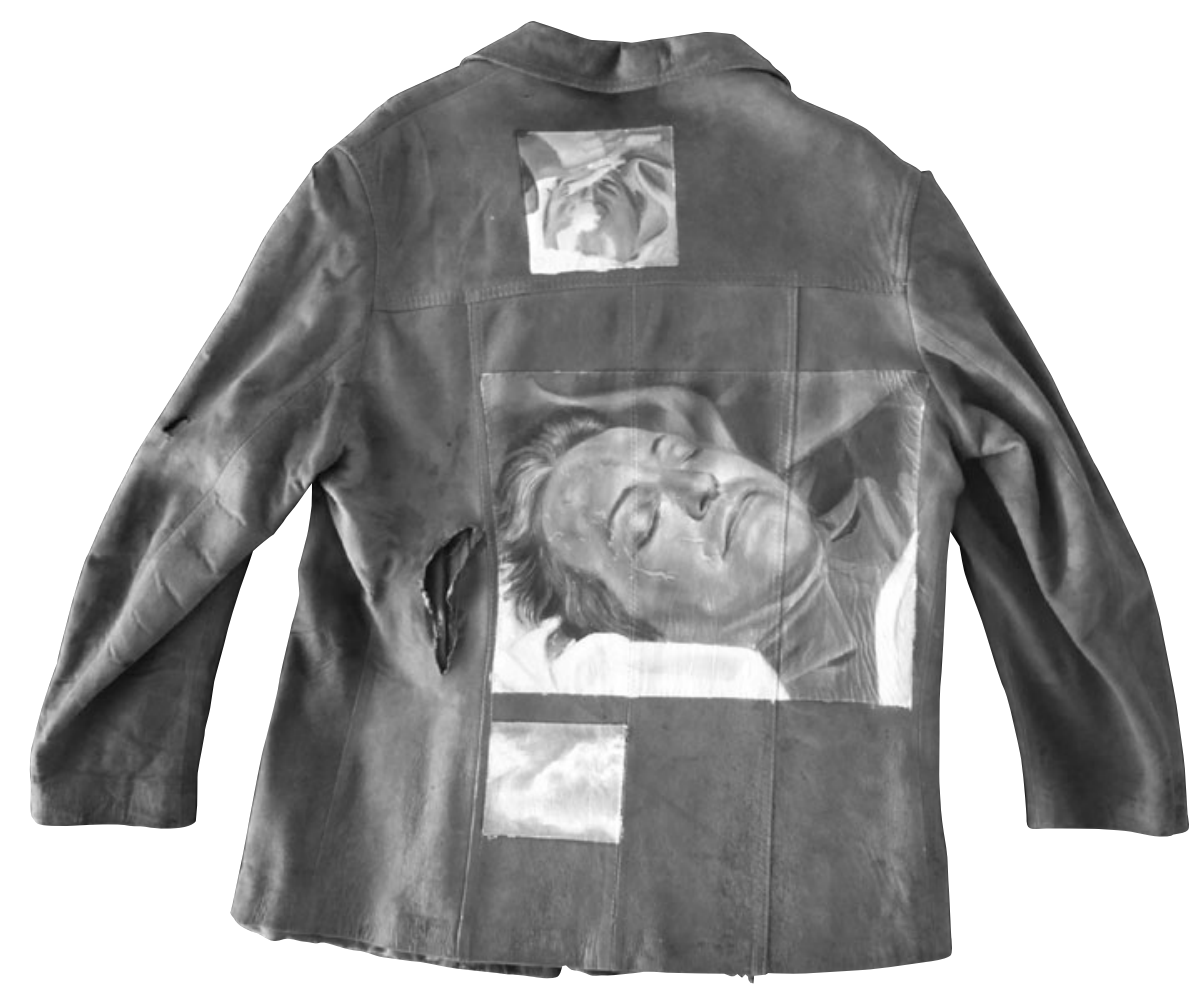

FIGURA 2. Chamarra/retrato de Javier Campos Cabello antes de la intervención (Fotografía: Miriam Limón Gallegos, 2011; cortesía: Museo de Arte de Zapopan, Jalisco, México). 
Campos Cabello (Figura 2), cuya finalidad consistía en comprender las características conceptuales de la pieza y su valoración. Con ello se consiguió saber que la chamarra originalmente perteneció a Campos Cabello, y que tras su muerte, en mayo de 1994, fuera parte de un homenaje, consistente en dedicarle un altar de muertos en las instalaciones del Instituto Cultural Cabañas (ICC). Fue entonces que Lozano le solicitó a Martha Pacheco, íntima amiga del artista que en ese tiempo trabajaba en su taller, hiciera algo "especial" para depositarlo en el altar. Pacheco tuvo la idea de realizar el retrato usando un peculiar soporte: una chamarra de gamuza que el pintor había usado con regularidad. La imagen plasmada se basó en las fotografías tomadas durante el funeral, que retrataban "la paz interior que reflejaba" el artista en su último lecho (Lozano 2012). La pieza, que se montó en el altar del 2 de noviembre de 1995, la usó en un par de ocasiones Lozano, y posteriormente permaneció en una vitrina en la casa de su dueña. No volvió a plantearse la posibilidad de su exhibición pública sino hasta 2011.

Sobre la restauración de la pieza se planteó que para integrar la composición plástica del retrato era necesaria la realización de una reintegración cromática, ya que las pérdidas de color modificaban el aspecto y no permitían entender la imagen, pues mayoritariamente se encontraban en el rostro de la persona del retrato mortuorio (Figura 3).

Posteriormente se entrevistó a Pacheco, quien, a pesar de haber tenido una relación muy cercana con el artista fallecido, no había tenido contacto con la pieza sino hasta entonces. Sin embargo, al enterarse de la problemática de conservación de su pieza, Pacheco coincidió con Lozano que era necesario la reintegración en las áreas faltantes no sólo para la exhibición, sino también para el futuro, ya que la imagen, seriamente alterada, "le restaba respeto a Javier" (Lozano 2012).

De estos diálogos surgió una principal interrogante: ¿quién intervendría la obra? Inicialmente se tomó en consideración que la artista, por tratarse de un objeto que conocía en su factura y valoraba personalmente, querría restituirla. Sin embargo, se juzgó que ello no se trataría propiamente de una restauración. Por tanto, las restauradoras hablaron con ambas partes —artista y coleccionista-, y plantearon dos rutas, ambas observantes tanto del reconocimiento de los valores presentes en el objeto como de una metodología profesional de intervención en materia de restauración, a seguir. La primera trayectoria era que las restauradoras reintegraran las zonas de faltantes, siguiendo una metodología de registro gráfico y documental de la pieza, así como el uso de un sistema de reintegración y materiales que permitieran la integración de las lagunas, las que, si se deseaba, podrían eliminarse en algún momento; la segunda era que la propia artista, asesorada por las restauradoras, que realizarían el registro y la ayudarían con la selección de materiales, aplicara color sobre las zonas de pérdida. Ante este panorama,
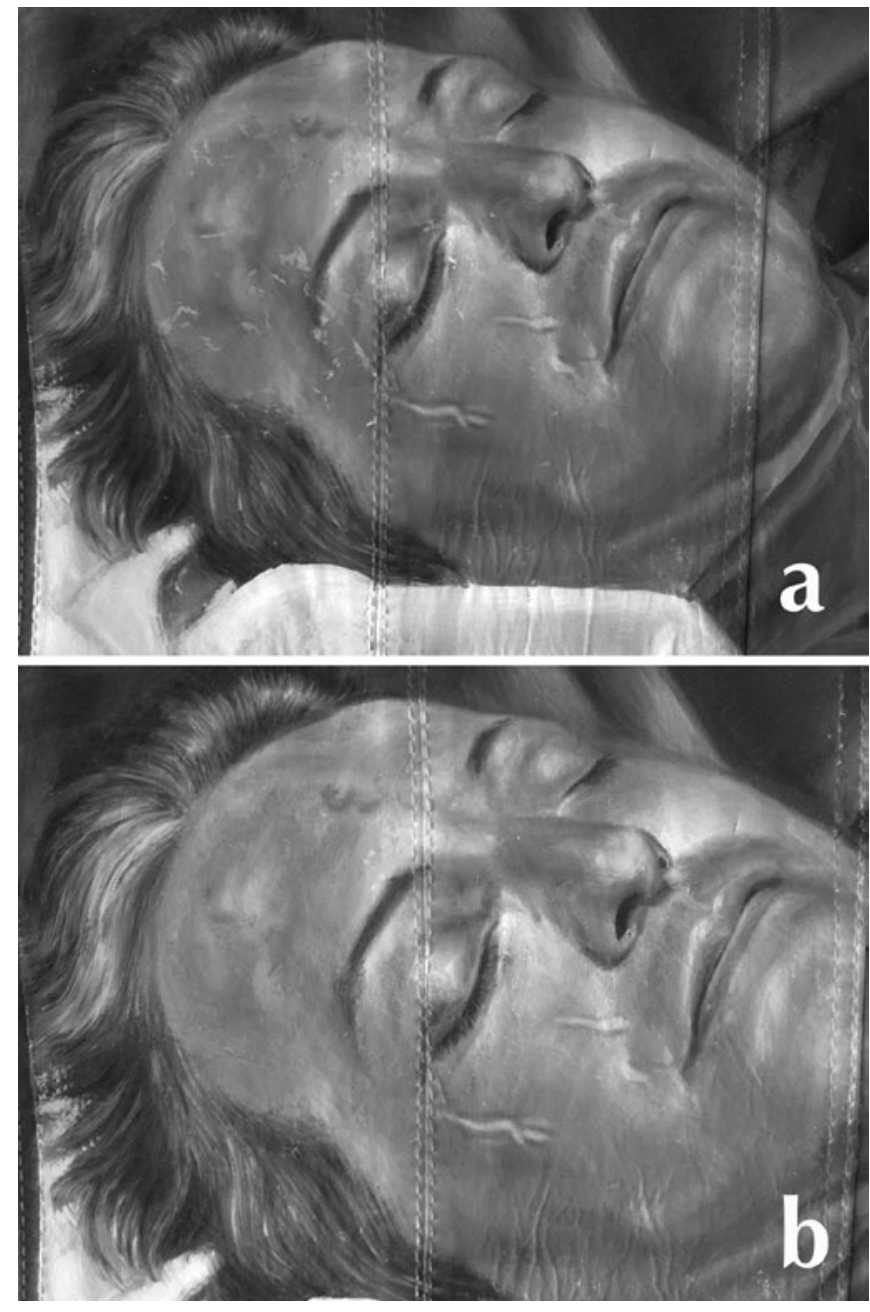

FIGURA 3. a) Detalle de la zona del rostro de la Chamarra/retrato antes de la intervención; se observan las pérdidas de la capa pictórica en las zonas de frente, ojos y mejillas que interrumpen áreas de luz y las lágrimas. b) Detalle de la pieza después de los procesos de restauración (Fotografía: Miriam Limón Gallegos, 2011; cortesía: Museo de Arte de Zapopan, Jalisco, México).

tanto la dueña como la artista preguntaron cuál sería la opción más adecuada para la intervención, y obtuvieron como respuesta no sólo que ambas opciones eran válidas sino que se respetaría cualquiera de ellas.

La decisión consensuada fue que las restauradoras llevaran a cabo la intervención: se creyó que era mejor que las especialistas realizaran ese proceso, ya que además de contar con los conocimientos teóricos y prácticos, entendían la relación entre la materia, el mensaje y la carga emocional. Así fue posible realizar la intervención; primero, colocando un soporte plano rígido en el interior de la chamarra/retrato, para facilitar su manejo y evitar futuras deformaciones; más adelante se realizó una limpieza superficial sobre la capa pictórica, ya que la suciedad adherida alteraba los colores, más evidentemente sobre los tonos claros, y finalmente la reintegración de color sobre las zonas de pérdida, usando gouache, aplicado a modo de nutrido. Cabe mencionar que la selección 
del material se realizó debido a pruebas de solubilidad previas, las cuales demostraban que las escamas de la capa pictórica eran susceptibles a solventes no polares, causando deformaciones e intensificando los desprendimientos; de ahí que se decidiera optar por un material que empleara como medio un solvente polar que además se integrara con las características de brillo y saturación de la superficie pictórica, considerando el gouache como la opción que cumplía con estos requerimientos, aparte de que, debido a las texturas de las áreas circundantes, el sistema de puntillismo hacía posible integrar el color a las zonas de pérdida (Roja de la Roja 1999: 35).

Una vez intervenida, la pieza se exhibió durante la inauguración y toda la estadía de la exhibición, con lo que la dueña y la artista quedaron conformes con el resultado (Figura 4).

\section{Conclusiones}

Restaurar siempre implica decisiones complejas. En este caso no sólo se logró un conocimiento profundo de la obra sino que también se reconoció el derecho del artista a decidir sobre la misma, ya que ella misma, como generadora de la idea, podría haber efectuado la intervención a través de la materia. Por ello, planteamos que el

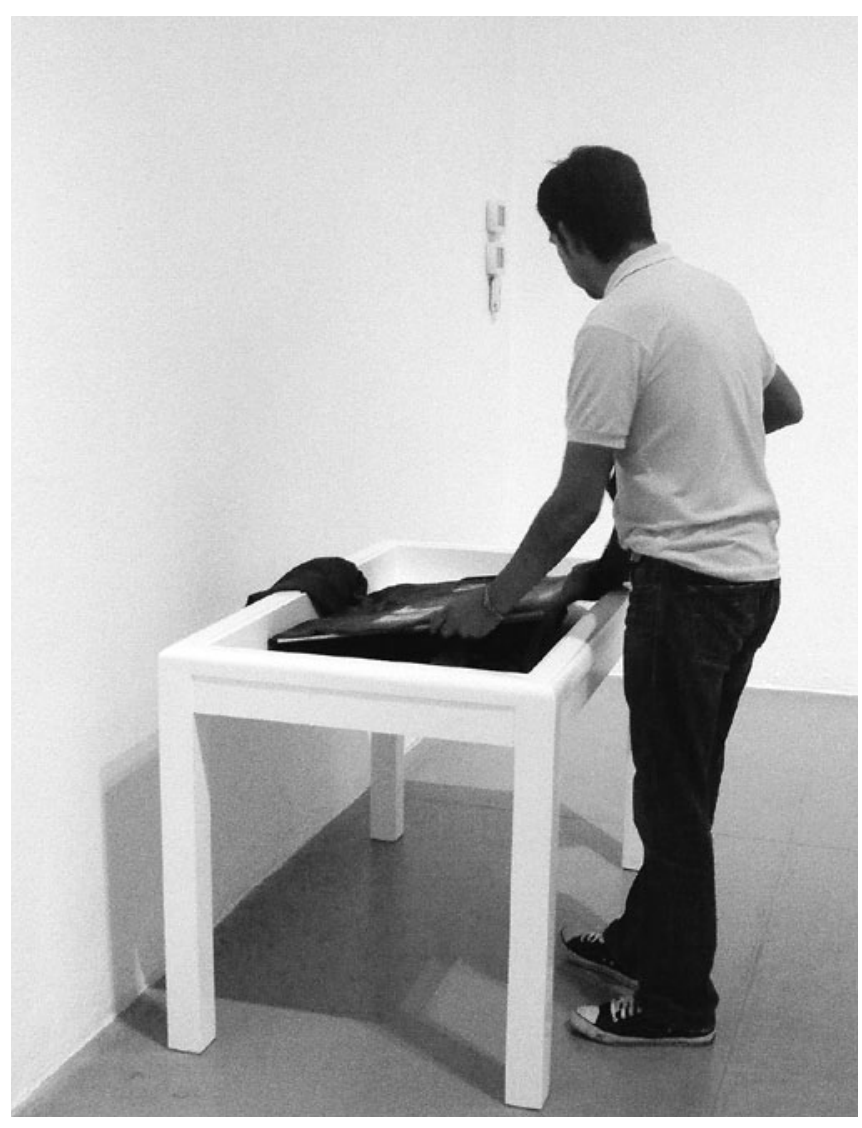

FIGURA 4. Montaje de la pieza en la vitrina de exhibición, antes de la inauguración de la exposición (Fotografía: Miriam Limón Gallegos, 2011; Cortesía: Museo de Arte de Zapopan, Jalisco, México). acercamiento al arte contemporáneo supone un esquema diferente y abierto, donde cada caso es único y plantea soluciones diferentes: mientras en el arte tradicional una intervención de restauración se propone tras realizar estudios de carácter historiográfico y científico, la propuesta de intervención para el arte contemporáneo se convierte - en cambio - en una toma de decisiones que puede llevar incluso a no intervenir materialmente el objeto, y aplicar solamente acciones de conservación preventiva, dejando en algunos casos que éste se degrade, en busca únicamente de la permanencia del concepto ideado por el artista. Así, para cualquier objeto enmarcado en el arte contemporáneo la documentación no sólo es primordial sino se convierte, en varios casos, en la única acción posible de conservación.

Se hace claro que el restaurador no puede ser un elemento aislado del grupo social en el que se desarrollan las obras, que sólo plantea las rutas críticas e interviene el objeto: también es necesario que guíe el diálogo entre los involucrados que, como él, buscan estabilizar los objetos, y participar activamente en el variable ambiente de apreciación conceptual en que se inserta la obra: como lo menciona Heinz Althöfer (2003:12), "una mentalidad limitada a lo puramente técnico constituye el punto de partida seguro para cometer errores y equivocaciones".

\section{Referencias}

Althöfer, Heinz (ed.)

2003 Restauración de pintura contemporánea. Tendencias, materiales, técnicas, Madrid, Akal.

Barbero Encinas, Juan Carlos

2008 Fondo y figura. El sentido de la restauración en el arte contemporáneo, Madrid, Polifemo.

Cauquelin, Anne

2002 El arte contemporáneo, México, Publicaciones Cruz O. Llamas Pacheco, Rosario

2006 "Investigar para enseñar o cómo profundizar en el conocimiento de la disciplina de la conservación del arte no convencional", en XVI Congreso Internacional de Conservación y Restauración de Bienes Culturales, Valencia, Universitat Politècnica de València.

2009 Conservar y restaurar el arte contemporáneo. Un campo abierto a la investigación, Valencia, Universitat Politècnica de València.

Lozano, Alicia

2012 "Realización de la Chamarra/retrato de Javier Campos Cabello", entrevista realizada el 8 de enero de 2012.

Mata Delgado, Ana Lizeth y Karen Landa Elorduy

2011 "La intervención del artista en la restauración del arte contemporáneo", Intervención. Revista Internacional de Conservación, Restauración y Museología (3), 74-79.

Muñoz Viñas, Salvador

2003 Teoría contemporánea de la restauración, Madrid, Síntesis. 
Roja de la Roja, Juan Manuel

1999 "Sistema de reintegración cromática asistido por medios transferibles obtenidos por procedimientos fotomecánicos. Aplicación en la restauración de pintura de caballete", tesis de Doctorado en Pintura y Restauración, Madrid, Universidad Complutense de Madrid.

\section{Resumen}

Durante las decisiones tomadas en torno de piezas pertenecientes al arte contemporáneo es necesario que el restaurador actúe como un intermediario con el fin de involucrar a las partes relacionadas con los objetos, y de este modo conocer los valores que rodean a las piezas para, así, obtener una intervención integral. En este artículo se muestran las consideraciones y reflexiones realizadas durante la intervención de la pieza Chamarra/ retrato de Javier Campos Cabello en el marco de la exhibición Martha Pacheco. Exposición antológica, realizada en el Museo de Arte de Zapopan (MAZ), en Zapopan, Jalisco, México.

\section{Palabras clave}

Toma de decisiones, arte contemporáneo, artista, coleccionista, conservador-restaurador.
Scicolone, Giovanna C.

2002 Restauración de la pintura contemporánea. De las técnicas de intervención tradicionales a las nuevas metodologías, Sevilla, Nerea.

\section{Abstract}

When decisions are taken regarding contemporary art, the conservator-restorer must play an intermediary role to involve the stakeholders related to the object, and, in this way, recognise the values that the work of art holds for different actors, an approach that actually supports an integral intervention. This paper shows the considerations and reflections during the intervention of Jacket/portrait of Javier Campos Cabello, an art piece exhibited at Martha Pacheco. Exposición Antológica, in the Museo de Arte de Zapopan (MAZ), Jalisco, Mexico.

\section{Key words}

Decision-making, contemporary art, artist, collector, conservator-restorer.

Título en inglés: The restorer as an intermediary in the intervention of contemporary art: the decision-making process 